\title{
Bi-tonal Architectural Narratives: Design Expressions in Manga
}

\author{
Marc Aurel Schnabel \\ School of Architecture, The Chinese University of Hong Kong, Hong Kong \\ marcaurel@cuhk.edu.hk
}

\begin{abstract}
Bi-tonal, or black and white architectural renderings are a common medium to present an architectural design that goes beyond factual representations of dimensions of length, width and height, materials, structure or technical details. They aim to engage their audience with visual aspects as well as emotional reactions. An architectural presentation that is based on a story-telling approach, akin to comic or manga style, adds intangible expressions, which allows communicating key elements as well as concepts, development and overall aspects of a design easily to laypersons and professional alike. This paper presents how designers develop a narrative of their architectural proposals using a bi-tonal visualization and story-telling methodology that communicates design to a wider audience.
\end{abstract}

Keywords: Digital manga, architectural depiction, visual communication, story-telling.

\section{Introduction}

Sketches and renderings are the most common methodology which architects use to communicate their design and proposals to peers, clients, consultants, contractors, or the general public. Over the past two to three decades computational tools allow architects to generate depictions that offer a communication of the designs that is appears to be reality via photo-realistic rendering, video walkthroughs, or similar computer generated depictions. Despite these advances, there is a great value in the abstract and simplified visualization that hand sketches, black and white $(\mathrm{b} / \mathrm{w})$, or bi-tonal images offer. Technical drawings or sophisticated renderings not always allow professionals or laypersons to understand the complex facets and various elements of an architectural design. Hence the study proposes a particular form of representation that supplements factual and technical drawings: digitally generated, bi-tonal, nonphotorealistic images of architectural design that offer an easy accessible realm for communicating architecture. The genre of Japanese comics, called manga, is related to conventional architectural drafting with their $\mathrm{b} / \mathrm{w}$ patterns and representations. A manga artist lays multifarious screens to express different semantics building up each image to match the overall narrative of the story. Hereby the narrative offers a deeper engagement with the image by both the artist and the reader, while offering at the same time an enhanced legibility and appreciation by the general public. This paper 
discusses how designers generate and develop their ideas using bi-tonal architectural depictions of key elements of their designs and how they develop narratives to communicate them to a larger audience.

\section{Conventional and Digital Manga}

Despite its popularity there is no automated process to generate manga images and the task of drawing a series of images is rather tedious, time- and labor intensive, especially if the image is composed of large variety of details and elements, which is particular true for architectural depictions. Yet the way of narrating a story through a series of key images is a widely used and well understood methodology to communicate factual, fictive, as well as intangible elements and feelings.

The typical conventional steps for producing one manga image-frame are: First, artists decide the perspective and roughly sketch the major structure if the scene with pencils. Next, they finalize the drawing with ink. With the precise lines in place, artists then begin the screening procedure. Based on the inked lines, manga artist usually select appropriate pre-print screen sheets to fill regions in order to express shading, tone, texture, or atmosphere. The selected screen paper, which is semi-transparent with pre-printed patterns, is then overlaid on each of the target regions. The artist usually uses a knife to carefully carve out screen paper along the boundary and paste it on the target region. The image is ready when all the regions are overlaid with selected screens.
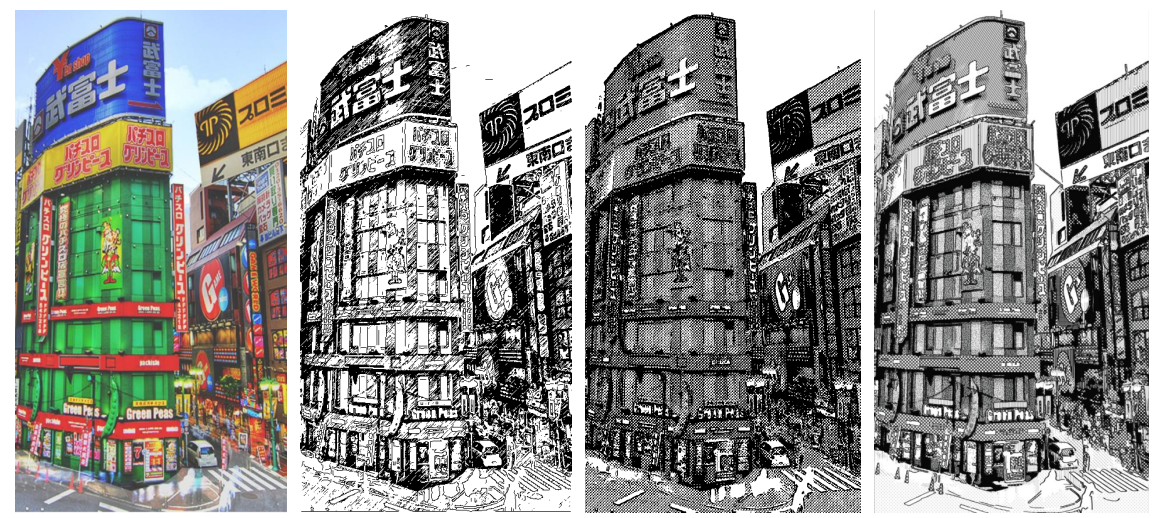

Fig. 1. From left: Original image and three digitally converted bi-tonal solutions: hatching, halftone, and Manga-Me

Only recently a system that automatically draws bi-tonal depictions using manga techniques was developed [1]. Although there are numerous tools available to convert digitally any input image, sketch or drawing into a bi-tonal manga picture, often the generated results does not look like manga. Qu et al's [1] system automatically generates a result that has the same quality as a conventionally produced image, yet with a fraction of time and costs (Figure 1). A simplified version of this system, Mange-Me, is an iPhone-application [2] that allows users to generate a depiction 
using the distinct expression of manga style visual communication. The system consists of two major components, screening and line drawing, which are similar to the conventional manga production described above. The line drawing includes an importance model that ranks each line, allowing architects to control the detail level of lines needed in their architectural manga. For the screening process, the system aims to automate the screen selection process allowing richness preservation and style consistency to conventional manga by utilizing the solution [3] that automatically selects appropriate bi-tonal screens to represent or fill up different regions in the input image or photograph.

\section{Bi-tonal Architecture Depiction}

Photorealistic visualizations of architectural design are the established method of communicating architectural ideas, concepts or existing buildings. Hereby the objective is to generate a highly realistic and factual representation of the designs. The quality of these renderings is so high that it is often impossible to distinguish them from real photographs and the illusion is nearly perfect. However, architectural spatial concepts such as geometry/composition, threshold, proportion/scale, circulation/duree, light/shadow and tectonics are lost in the colourful well-rendered details and images. In conventional sketching these architectural elements, properties, functions, or materials are either represented by standardized screens, hatches, line-types, styles, and -widths, or by the personal expressions of the architect. In the abstract representation of the sketch architectural spatial concept and other non-factual elements can be highlighted clearer. The above presented automated methodology of generating bi-tonal images allows for the development of a new generation of architectural design communication that reaches beyond graphical standard visualizations that include among others intangible elements and expressions.

Conventional rendering or a series of sketches missing a crucial element in their communication: the narrative. A collection of images, collages, diagrams, photographs and rendered images do not provide the reader any clue about how the design comes about, how it blends into the urban environment, how the building functions, and especially how emotions, atmospheres or expressions relate to the design. Standardized depictions provide the factual outlook, practicality, and buildability of the design only, but not a narrative that engages the reader with the design akin to a movie or novel. On the other hand, bi-tonal, non-photorealistic depictions are increasingly becoming popular around the world as a mean of expression and story-telling because of its distinct graphical quality with its elegant use of rich set of screens, tidy and fine drawing styles of b/w drawings, and its engaging ways of its narrative [4].

In architectural design for example, the commonly used software SketchUp ${ }^{\mathrm{TM}}$ provides a few additional options that change the line style of a model, giving it a unique raw outlook, mimicking hand-sketching, in order to portray architecture in a simple outlook with just its basic geometric elements. In this way many digital sketches are produced.

The Bjarke Ingels Group (BIG) goes a step further. This group of architects, designers, and theorists operate within the fields of architecture, urbanism, research and development. Based on the methodology of comics, their book 'YES is More' [5] 
introduces their works through this very format. It is a playful gesture to make architecture accessible and less formal. BIG ads speech bubbles and overlaid diagrams to their architectural designs, making them appear to be alive and talking to the reader. Although most images are simple photographs, yet the overall style is novel to how they convey architectural ideas and designs to the general public and professionals alike. Architectural manga as presented here goes even further to give architectural representations an enhanced immersion that goes beyond conventional architectural depiction and communication by using a bi-tonal narrative akin to manga.

\section{Bi-tonal Architectural Narrative}

In order to test how young designers, who are all familiar with the genre of manga are able to develop their own narrative of an existing, yet new building to them, a cohort of fifty second year undergraduate- and thirty first year master of architecture students were asked to generate an architectural narrative using the above presented smartphone application. The aim was to introduce the newly opened building of their School of Architecture (AIT), at the Chinese University of Hong Kong (CUHK), to their peers. Alternatively, the students could capture a particular urban setting of a high density and vibrancy neighborhood in Hong Kong, called Mongkok. Students then took their selection of converted bi-tonal images and collaged them into a comic narrative on maximal two pages by using either typical manga page lay-out templates (predefined subdivisions and image frames of pages) or their own generated designs to generate a short architectural story. The pages were uploaded onto a social network group [6] for comments dissemination and evaluation among a larger audience. This not only inspired other students, but also generated variations and sequels to emerge.

Figure 2 show the initial representative outcomes of the above described mangatask. The outcome was promising as students were able to easily utilize the unique quality and characteristic of manga to narrate a certain story or journey that is has the architecture as its protagonist. These examples act as initial framework of how designers use the medium to develop an architectural manga.
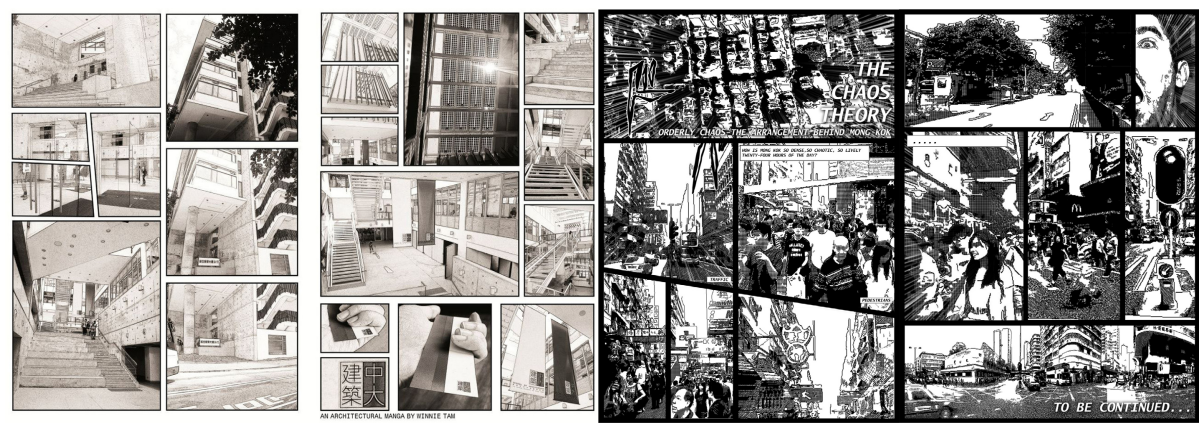

Fig. 2. Left. Walkthrough by Winnie Tam portraying her personal story of the AIT-building. Right: Motion supported manga by Kenton Sin: it depicts the seemingly chaotic arrangement of the urban setting and their impact on humans' movements through the streets of Mongkok 
The above descried method resulted in different understandings and reflections of the chosen building or urban setting. In conventional analyses designers collect a variety of data that are related to the site or building, though these gathered findings are often not used any further in the following design activities.

Students selected their own topics for their personal narratives and then generate a comic-strip to reinforce the structure of their narratives. However, by developing a story, the plot of a narrative is comprised of a series of "things that characters do, feel, think, or say" [7]. Hereby each of the events is an important component to the outcome of the story. These components have to be selected carefully, because a simple list of events or incidents, and in this case, depictions alone do not establish a plot. The portrayed architectural context, the building, and its details must be "significant events" rather than a simple series of images or details that happen. The exercise reviled that students understood the essence of an image based story and were only sometimes confused by the difference between a series of events or details that occur at their site or can be found at the building.

\section{$5 \quad$ Manga Design Expressions}

In a next step of reacting architecturally to their findings students were asked to generate their own designs based on their initial comic strip. Keeping the elements of a narrative not only in mind but also as their influential design parameter students were then given the freedom to continue working with the manga application at any stage of their next following architectural design studio with the same sites. Without going into further details of the design studio, this paper focuses on the phases at which the manga application was used and in which way. Details of the overall design studio and its process can be found at the social network websites of CAAD@CUHK [6] and Ornaments and Patterns [8].

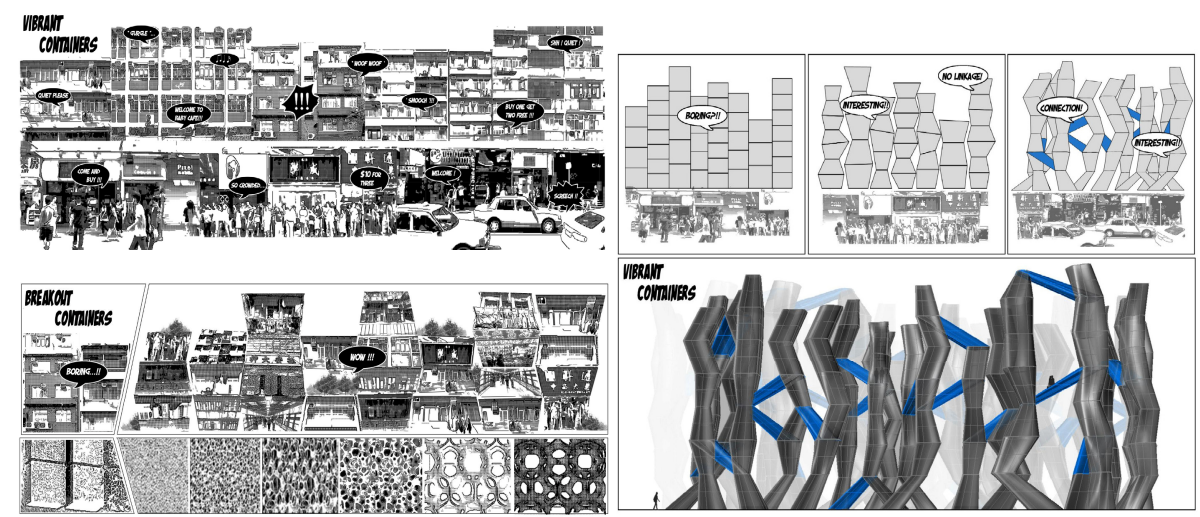

Fig. 3. Design by Anita Au: Form generated from the studies of urban context 


\subsection{Form}

The manga presented in Figure 3 demonstrates how the application gives the student a different perspective in her studies of the urban fabrics. By removing the colours of the original scene, the application enhanced the overall patterns of the urban setting and made them more distinct. The student made use of this by basing her further design development on the found patterns. The student used micro and macro elements of her manga story as parameters to generate her final proposals. There is a direct connection of the found parameters, events, and items of the initial narrative up to the final presentation.

\subsection{Façade}

Figure 4 shows how a student used a very similar visual study technique to generate a façade that developed it's dynamic from the elevations of the urban elevations. By extracting, outlining and then overlaying the layers of patterns, a unique façade system that can blend into the seemingly chaotic Hong Kong urban setting is created. This is possible due to the monotonous yet rich screening of the images generated by the manga application. The student made use of the existing façade patterns, which were emphasized by the translation of site-photos, to generate a design that is closely related to the urban vertical landscape, yet its original novel interpretation of a high-rise façade.

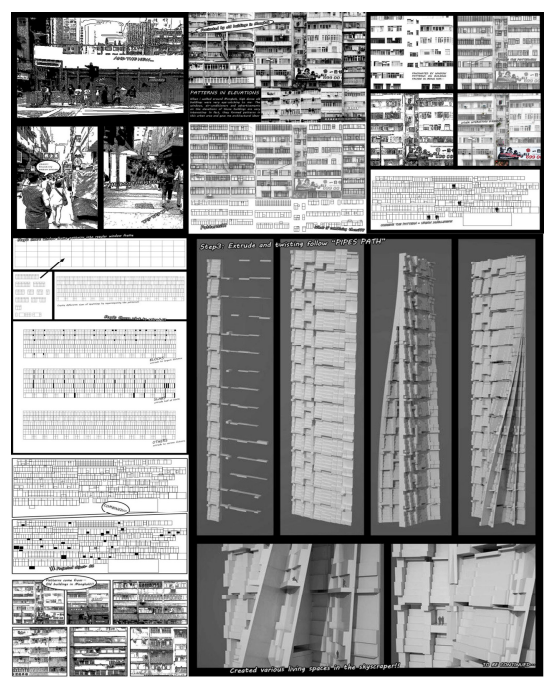

Fig. 4. Design by Esther Lam: Façade studies using the Manga App as base for her design

\subsection{Pattern}

The application provides great opportunity and inspiration which allowed students to use them as a generator of abstraction and pattern finder. The following author fully utilized the pattern that was visualised from the manga-generated image as a source of 
inspiration for her design (Figure 5). The interesting aspect of this abstraction is how an original 3D space was flattened into a 2D image and then re-interpreted threedimensionally. This has a strong relationship to the working methods of architects, who read 2D depictions, such as plan, section, or elevations, three-dimensionally in order to understand the depicted space.

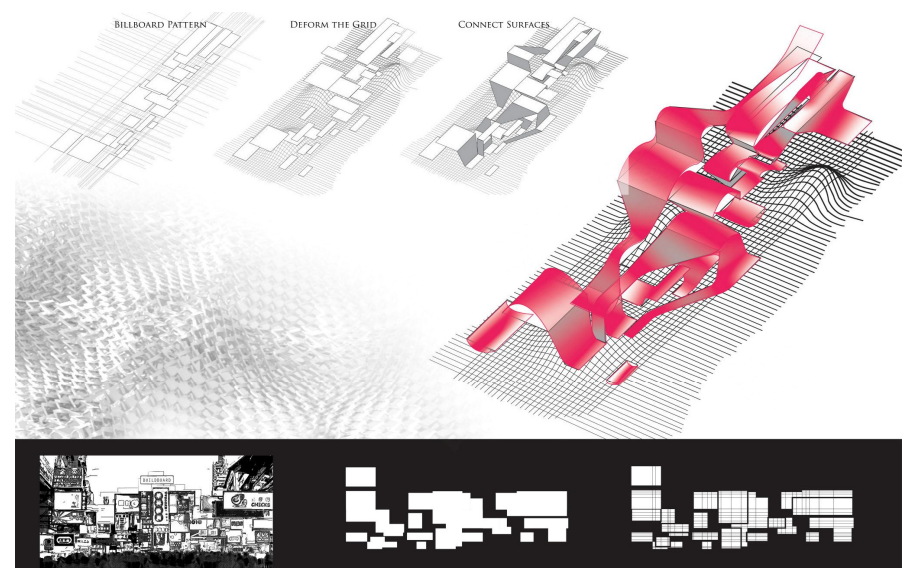

Fig. 5. Design by V. Ou-Yang: Pattern developed from images created by the Manga App

\section{$5.4 \quad$ Narration}

Students used the expression of manga to present their design in different ways. Instead of plan, section and elevations, the development of their design and its details became the central element of their design communication. Hereby they used not only

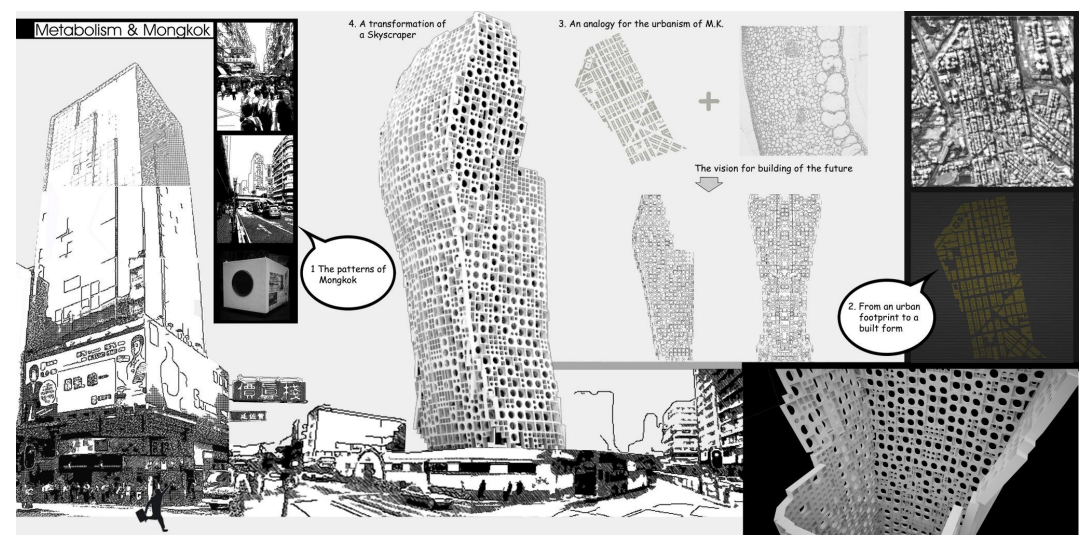

Fig. 6. Design by Leo Yu: Using manga as basis to narrate his final design 
a bi-tonal representation of their design, but transferred their experiences gained through the manga methodology to put a narrative into their design communication. In the student's work of Figure 6 a plot of the design is developed that takes the reader through the building in macro- and micro scale. The building or part of it by being the protagonist of the story and the reader becomes both, the observer of the story as well as the antagonist, having to react to the design and its urban context.

\subsection{Presentation}

The clear line drawing and simple screening of manga allows designers to use this method as a tool for their final design presentation. The visual communication of manga is a very simple and clean composition that brings visually together the various aspects of the design proposal. At the same time students included a narrative of how the design is formed and how it is related to the site context (Figure 7). Akin to a complete plot the author made use of his narrative as a way of presenting and expressing verbally his design. Herby he added elements of sound, drama and amusement to his voice allowing the audience to engage more direct with his proposal.

\subsection{Methodology}

This student took up the challenge of using Manga as the main design methodology throughout the whole process instead of just using it only in one part or as visual

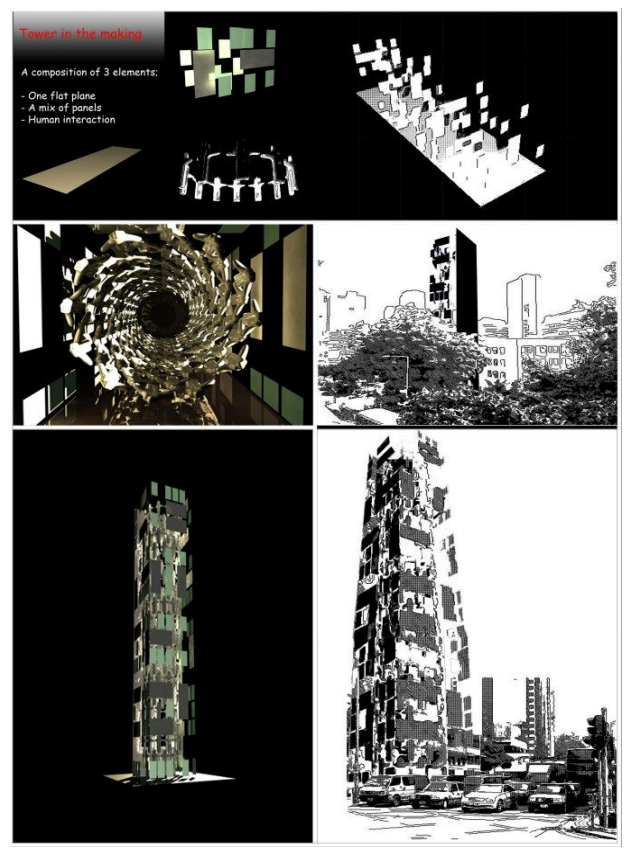

Fig. 7. Design by Mark Choi: Using site images created from manga apps for final design 
communication tool (Figure 8). From site narrative to next following initial massingand form-exploration all the way to the final presentation, she generated a design that springs off from her site-plot, fitting the character of lines and hatches, as well as reactive to the site context. Hereby she was able to propose a solution that presents the several possibilities of the distinct bi-tonal style and intersected tool and design objective.

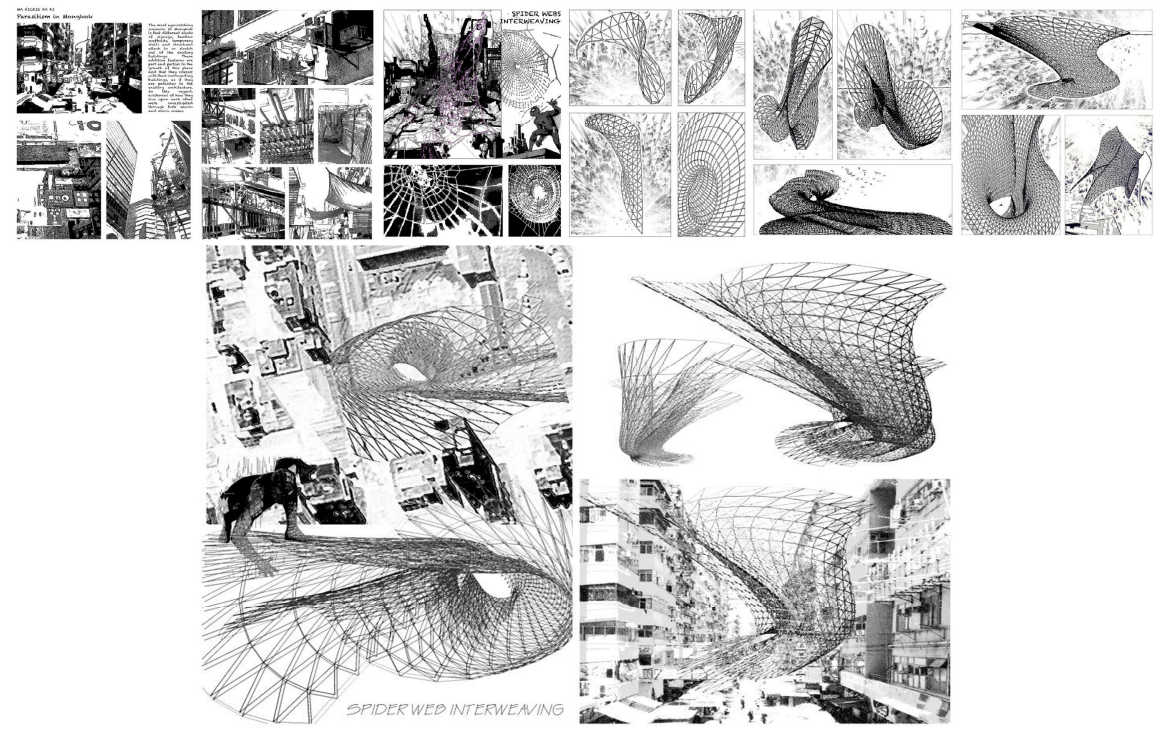

Fig. 8. Design by V. Ka: Using manga throughout her design process

\section{Conclusion}

This research has shown that designers not only develop with ease architectural narratives but also transformed the genre of manga to a novel direction that used architecture as the protagonist. Hereby the designers made use of the characteristic bi-tonal representation to aid them in their design generation, expression and communication. The manga-style depiction and narrative provided the designers an additional pointof-view to explore the site context and various architectural elements. The methodology of generating a narrative contributes to the overall design, whereby the plot of a narrative is comprised of a series of the events that are important to the outcome of the overall architectural design. A typical architectural representation of plan, section and elevations does not from a yet storyline that engages readers in other than factual elements of the architectural design. The depicted design elements of the architectural manga are crucial parts that relate to each other factual and emotional. In the above discussed proposals students generated comic-strips that are based on their own storyline of their designs, while the manga technique allowed them to efficiently and conveniently communicate their key elements to professionals and lay-persons. 
Hereby the expressions of manga are expanded to carry rich and meaningful content that merges architectural sketching, digital drafting and popular cultural media.

Visualization greatly facilitates the understanding and efficient reproduction of both basic and complex architectural design issues. Manga allows subsequently addressing common challenges faced by commonly by architects: low understandability and poor reproducibility of complex design solutions. The here presented outcomes relate and are reflected in other areas of science and technology [9].

The bi-tonal architectural narrative can also be used as an instrument of architectural education, whereby the manga-based representations of designs can facilitate learning. They can be a semiotic resource in learning and help learners to see factual relationships together with their projected, intangible elements of the presented designs $[10,11]$. While social networks offer a larger dissemination, evaluation and commenting they also facilitate innovative communications and further development of the narratives. With its distinct depiction of architecture elements, bi-tonal graphical depiction provides an easier access of how designs are generated and developed. Moreover, the hatching details allow students to learn how to do hand sketching, which pattern or hatching generates a certain outcome. Students learn how within a sketch color, texture and meaning are translated to generate specific characteristics of their designs. This 'reverse engineering' of sketching has the potential to blend over the education of digital and analogue design communication and expression in architectural design [12].

In the next steps of this research cinematic issues as presented by Calderon et al [13] or $\mathrm{Ng}$ et al [14], are explored and a tool for architectural animations based on bitonal renderings developed. Further research has to be done to improve the workflow elements to aid the designer in the process of generating a narrative, including text, special visual effects, lay-options, and online sharing and publishing.

Acknowledgements. The author acknowledges the research that lead to the development of the software 'manga-me' \{http://manga-me.tk\} by T. T. Wong, P-A. Heng and Y. Qu., CUHK. Students of the 2012 BSSc(AS) $2^{\text {nd }}$ Year and the MArch1 cohort at CUHK and various online participants of the two Facebook Groups are thanked for their voluntary participations. This project was funded by a CUHK Direct Grant.

\section{References}

1. Qu, Y., Pang, W.-M., Wong, T.T., Heng, P.-A.: Richness Preserving Manga Screening. ACM Transactions on Graphics 27(5), 155:1-155:8 (2008)

2. Manga_Me, iPhone Application Computer Science and Engineering Department, CUHK, http: / / www . manga-me.tk

3. Schnabel, M.A., Qu, Y.: Digital Manga Depiction. In: Herr, C.M., Gu, N., Roudavski, S., Schnabel, M.A. (eds.) Circuit Bending, Breaking and Mending: Proceedings of the 16th International Conference on Computer-Aided Architectural Design Research in Asia, Newcastle, Australia, pp. 741-750. Association for Computer-Aided Architectural Design (CAADRIA) (2011) 
4. Qu, Y., Schnabel, M.A.: Drawing Architecture using Manga Techniques. In: Dikbas, A., Ergen, A., Giritli, H. (eds.) Managing IT in Construction / Managing Construction for Tomorrow, CIB W78, pp. 567-576. CRC Press/Balkema, Istanbul, Turkey (2009)

5. Ingels, B.: Yes Is More: An Archicomic on Architectural Evolution, Bjarke Ingels Group. Evergreen, Taschen, UK (2009)

6. CAAD@CUHK, Facebook Group of School of Architecture, CUHK, http: / / www. facebook. com/groups / CAADATCUHK

7. Dibell, A.: Plot, Cincinnati, OH: Writer's Digest Books (1988)

8. Ornaments and Patterns, Facebook Group of School of Architecture, CUHK, http: / / www. facebook. com/groups / OandP

9. Journal of Visualized Experiments website, http://www. jove.com/about

10. Herbst, P., Chazan, D., Chen, C.-L., Chieu, V.-M., Weiss, M.: Using comics-based representations of teaching, and technology, to bring practice to teacher education courses. ZDM 43(1), 91-103 (2011)

11. Tatalovic, M.: Science comics as tools for science education and communication: a brief, exploratory study. Jcom 8(4), A02, 17 pages (2009)

12. Horvath, I., Rusak, Z., Duhovnik, J.: Computational methods to support sketching, reverse engineering, and optimization of shapes. Journal of Engineering Design 18(5), 391-394 (2007)

13. Calderon, C., Nyman, K., Worley, N.: The architectural cinematographer: creating architectural experiences in 3D real-time environments. International Journal of Architectural Computing 4(4), 71-90 (2006)

14. Ng, K., Schnabel, M.A., Kvan, T.: Architectural animation becomes alive: creating spatial narrative with spatial characters for animations. In: Bourdakis, V., Charitos, D. (eds.) Proceedings of the 24th eCAADe Conference, eCAADe, Volos, Greece, pp. 598-603 (2006) 